\title{
On the Implementation of Optimal Receivers for LFM Signals using Fractional Fourier Transform
}

\author{
Çag̃atay Candan \\ Middle East Technical University (METU) \\ Ankara, Turkey. \\ ccandan@metu.edu.tr
}

\begin{abstract}
We present a novel receiver structure for the detection and parameter estimation of linear frequency modulated signals. The proposed structure is based on the relations between the fractional Fourier transform and the ambiguity function. It has been shown that the optimal ML receiver, which is the peak detector in the ambiguity plane, can be implemented at a reduced search complexity with the proposed method. The proposed method uses two 1-dimensional search operations in two different fractional Fourier domains instead of a 2-dimensional search over the ambiguity plane. The performance of the method is illustrated with an example.
\end{abstract}

Index Terms-Pulse-Doppler coupling, LFM signals, Radar receivers

\section{INTRODUCTION}

Linear frequency modulated (LFM) waveforms are frequently used radar waveforms with well known advantages such as high range resolution and Doppler tolerance. A drawback of LFM signals is the coupling between range and Doppler estimates. It is known that when a correlation based receiver is used to detect LFM signals, the detected range suffers from an offset range which is proportional to the Doppler frequency of the incoming echo, [1]. In other words, a target may appear at a closer or at a further away range than its true position if the return from the target has a nonzero Doppler frequency. In the applications where the pulse duration is shorter than a few miliseconds, range-Doppler coupling has a negligible effect on the range estimation. In other applications where the pulse duration can be on the order of seconds, as in sonar applications, the offset due to coupling can be significant, [2].

In this paper we examine a receiver structure based on the cross-ambiguity function. We use the following definition for the cross-ambiguity function:

$$
\mathcal{A}_{r s}\left(\tau, f_{d}\right)=\int_{-\infty}^{\infty} r(t+\tau / 2) s^{*}(t-\tau / 2) e^{i 2 \pi f_{d} t} d t
$$

When $r(t)=s(t)$ the cross-ambiguity function reduces to the ambiguity function which is also called as the autoambiguity function. The magnitude square of ambiguity function, $\left|\mathcal{A}_{r s}\left(\tau, f_{d}\right)\right|^{2}$, is called ambiguity surface. It is known that the ambiguity surface is the ML detection statistics for the estimation of delay and Doppler frequency of the echo signal under white noise and flat fading channel conditions, [3]. In other words, an optimal ML estimator executes a two dimensional search for the peak location to estimate the delay and Doppler frequency of the target:

$$
\left[\widehat{\tau}, \widehat{f}_{d}\right]=\arg \max _{\tau, f d}\left|\mathcal{A}_{r s}\left(\tau, f_{d}\right)\right|^{2}
$$

The contours of equal height in the ambiguity plane indicate the locus of equally likely delay and Doppler frequency pairs. In conventional systems, the operation of the ML receiver is approximated with a filterbank of matched filters, [1], [3].

When LFM signals are used as radar waveforms, a characteristic feature of LFM signals can be used to reduce the dimensionality of the search in the ambiguity plane. A LFM signal with the chirp rate $k$ and duration $T$ is defined as $s(t)=e^{j \pi k t^{2}} \operatorname{rect}(t / T)$, where $\operatorname{rect}(t / T)=1$ when $t<|T| / 2$ and zero otherwise. For illustration purposes, the ambiguity surface of LFM signal with the chirp rate 0.6 and duration of 4 seconds is shown in Figure 1.

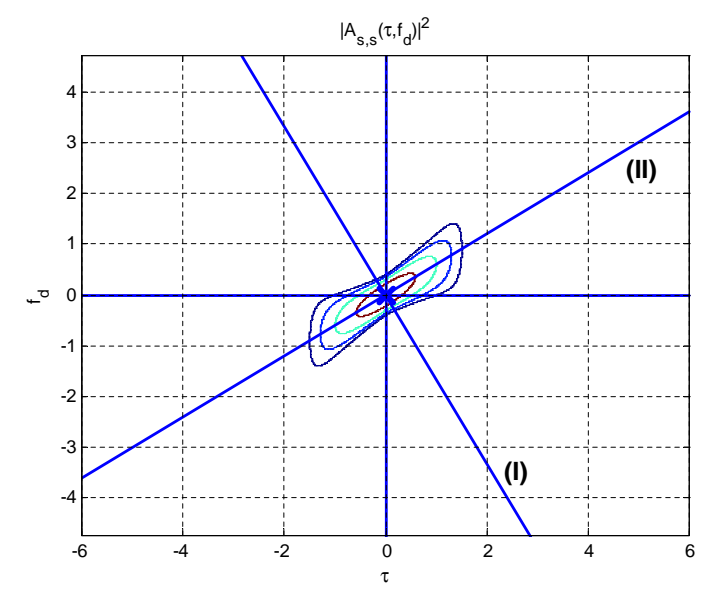

Fig. 1. Ambiguity surface for the LFM signal with $k=0.6$ and $T=4$.

For LFM signals with infinite duration, the ambiguity function reduces to a line impulse over its instantaneous frequency, $\mathcal{A}\left(\tau, f_{d}\right)=\delta\left(f_{d}-\tau k\right)$. For finite duration LFM signals, the signal is spread over the ambiguity plane, but its energy is concentrated along the instantaneous frequency line.

The conventional method to detect LFM signals is matched filtering. The output of the matched filter in the absence of noise is the cross-correlation between transmitted LFM signal and the received signal. The output is also equal to the slice of the cross-ambiguity function at $f_{d}=0, \mathcal{A}_{r, s}(\tau, 0)$. In other 
words, the conventional receiver evaluates the cross-ambiguity function along the line $f_{d}=0$.

Here we present an alternative receiver evaluating different slices of ambiguity function. We introduce a two stage detection process similar to the filterbank of method of the conventional receivers. In the proposed method, the first stage operates in the fractional domain which is orthogonal to the instantaneous frequency line of the chirp signal. The domain of the first stage is shown with line (I) in Figure 1. The second stage operates in the fractional domain which is aligned with the instantaneous frequency line as shown with line (II). 1dimensional search operations along the lines (I) and (II) are conducted sequentially to detect the peak in the ambiguity plane.

Methods similar to the one proposed in this paper can be found in the literature. Wang, Ozdemir and Akay independently proposed to use Radon-ambiguity transform to detect chirp signals, [4], [5], [6]. The results of Ozdemir [5] have been refined and proposed as a detection solution in [7]. More recently Radon ambiguity transform has been used to detect other chirp like signals in [8].

Here we present a solution for the joint estimation of range and Doppler frequency for LFM signals. The proposed method is similar to the one proposed in [7]. In [7], authors use multiple domains to detect the target range and Doppler frequency. Here, we use two domains as illustrated in Figure 1.

\section{Ambiguity FUnCtion AND FRACTIONAL FoURIER TRANSFORM}

The fractional Fourier transform generalizes the ordinary Fourier transform to a continuum of transforms. The definition of fractional Fourier transform is given as

$$
\mathcal{F}^{a}\{f\}\left(t_{a}\right)=A_{\phi} \int f(t) e^{i \pi\left(t_{a}^{2} \cot \phi-2 t_{a} t \csc \phi+t^{2} \cot \phi\right)} d t
$$

where $\phi=a \frac{\pi}{2}$ and $A_{\phi}$ is a unit norm constant depending on $\phi$, [9]. When $a=1$, the definition in (3) becomes identical to the definition of the ordinary Fourier transform. When $a \rightarrow 0$, the transform approaches identity transform, that is as $a \rightarrow$ $0, f_{a}(t) \rightarrow f(t)$ and the kernel of the transform approaches $\delta\left(t-t_{a}\right)$. For any value of $a, f_{a}\left(t_{a}\right)$ is a unitary transform of $f(t)$.

Fractional Fourier transform is intimately connected to the Wigner distribution and the ambiguity function. The relationship between Wigner distribution and fractional Fourier transform has been used extensively in time-frequency analysis, [10]. But its relationship with the ambiguity function is dispersed in the literature, [9], [6], [4] even though the central relation, which is instrumental for our purposes, appears in the classical text of Van Trees [3, page 310].

There are two fundamentally important results on the fractional Fourier transform. Fractional Fourier transform can localize a chirp signal to a delta function in a fractional domain. This property makes the transform useful in applications involving chirp signals such as free space propagation, lenswave interaction and LFM signal detection. The second result is related to the time-frequency distributions. It is well known that the fractional Fourier transformation of a function has a Wigner distribution which is the rotated version of the original function by a fractional of 90 degrees in clockwise direction, [9]. The rotation property also applies to the ambiguity function.

Next we examine the connection between fractional Fourier transform and ambiguity function. The following two properties are well known for the ambiguity function:

$$
\begin{aligned}
A_{r, s}(\tau, 0) & =\int_{-\infty}^{\infty} r(t) s^{*}(t-\tau) d t \\
\int_{-\infty}^{\infty}\left|\mathcal{A}_{r, s}\left(\tau, f_{d}\right)\right|^{2} d f_{d} & =\int_{-\infty}^{\infty}|r(t)|^{2}|s(t-\tau)|^{2} d t
\end{aligned}
$$

The first property is the cross-correlation property which has been mentioned earlier. The second property does not appear in classical texts, but appears in [11].

A brief proof for the second property can be given by the Parseval's relation for the conventional Fourier transform. For a fixed $\tau$, the ambiguity function can be viewed as the Fourier transform of the time function $r(t+\tau / 2) s^{*}(t-\tau / 2)$. When Parseval's relation is applied to this function, we immediately get (5).

The second property can be interpreted in three different ways: It can be interpreted as the area under the slice of ambiguity surface function for a fixed $\tau$. It can also be interpreted as the projection of $\left|\mathcal{A}_{r, s}\left(\tau, f_{d}\right)\right|^{2}$ to $\tau$ axis, or can be interpreted as the marginalization of the time-frequency correlation function $\left|\mathcal{A}_{r, s}\left(\tau, f_{d}\right)\right|^{2}$ over the variable $f_{d}$.

The fractional Fourier transform generalizes the relations given in (4) and (5) to the following forms:

$$
\begin{array}{r}
\mathcal{A}_{r, s}(\tau \cos \phi, \tau \sin \phi)=\int_{-\infty}^{\infty} r_{a}\left(t_{a}\right) s_{a}^{*}\left(t_{a}-\tau\right) d t \\
\int\left|\mathcal{A}_{r, s}(\tau \cos \phi-u \sin \phi, \tau \sin \phi+u \cos \phi)\right|^{2} d u= \\
\int_{-\infty}^{\infty}\left|r_{a}\left(t_{a}\right)\right|^{2}\left|s_{a}\left(t_{a}-\tau\right)\right|^{2} d t
\end{array}
$$

The equation (6) states that a radial slice of ambiguity function along the line $f_{d}=\tan (\phi) \tau$ can be calculated from cross-correlation of the ath domain fractional Fourier transforms of $r(t)$ and $s(t)$. Similarly equation (7) can be interpreted as the the projection of $\left|\mathcal{A}_{r, s}\left(\tau, f_{d}\right)\right|^{2}$ onto the line $f_{d}=\tan (\phi) \tau$. Note that when $\phi=0$ the generalized equations reduce to their special cases given in (4) and (5).

Proofs of the generalized relations can be based on the rotation property of fractional Fourier transform. It is known that the fractional Fourier transform rotates the ambiguity function as it rotates the Wigner distribution, [9]. When relations (4) and (5) are used on $r_{a}\left(t_{a}\right)$ and $s_{a}\left(t_{a}\right)$ (the $a$ th order fractional Fourier transformations of $r(t)$ and $s(t)$ ), the generalized results are obtained. The generalized relations have been proved in the literature independently by various authors. One can consult [5], [6] and [7] for the algebraic 
proofs of (6) and (7) ${ }^{1}$.

\section{PROPOSED RECEIVER}

The proposed receiver is described with an example. A LFM signal with the duration of 8 seconds and with the chirp rate of 0.6 is used as the transmit waveform. The transmitted signal is received at an unknown delay and unknown Doppler frequency offset. Figure 2 shows the real part of the transmitted and received signals.

$\mathrm{s}(\mathrm{t})$ : Transmitted Signal (Real Part)

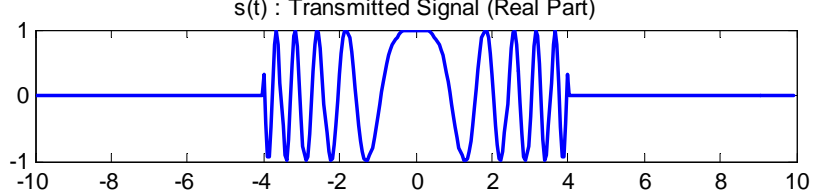

$r(t)$ : Received Signal (Real Part)

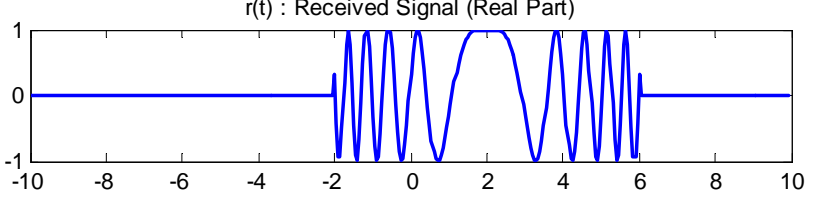

$A_{r, s}(\tau, 0):$ Matched Filter Output (Magnitude)

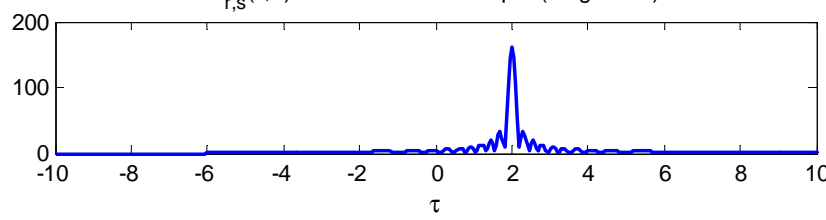

Fig. 2. Transmitted signal, received signal and the matched filter output. Signal is received at a delay of 2 seconds and has no Doppler shift.

In Figure 2, the received signal has a delay of 2 seconds with no Doppler frequency shift. Under these conditions matched filter produces the correct estimate of the signal delay.

In Figure 3, we observe the effect of range-Doppler coupling. In this figure, the received signal has the same delay, but it has a Doppler frequency shift of $0.6 \mathrm{~Hz}$. Under these conditions, the target has an apparent delay of 1 seconds when a matched filter based receiver is used. As noted before, unknown Doppler frequency shift affects the matched filter output and therefore a filterbank of receivers is needed to correctly estimate the signal delay and Doppler frequency.

Figure 4 shows the cross-ambiguity function of received signal and transmitted signal. The target is located at the point $(2,0.6)$ as shown with a cross. It is clear from this figure that a peak search in the cross - ambiguity function results can extract the true parameters of the target.

In this paper, we propose to make use of LFM signal characteristics to reduce the search dimensionality in the ambiguity plane. With the proposed method, the 2-dimensional search is reduced to two 1-dimensional search operations.

In the first stage of the search process, we calculate the projections of $\left|\mathcal{A}_{r, s}\left(\tau, f_{d}\right)\right|^{2}$ over the domain shown with (I).

${ }^{1}$ Note that [5] uses a different definition of the ambiguity function and a different choice of axis orientations. Therefore the relation derived in [5] looks different from the one presented here, but identical to ours in principle.

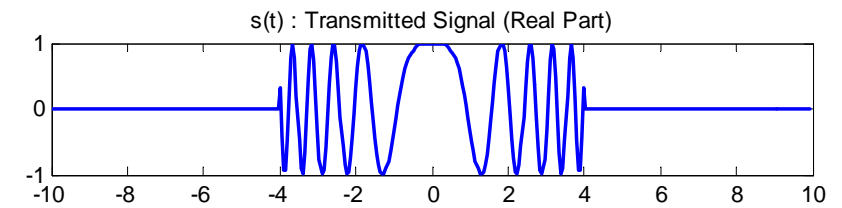

$r(t)$ : Received Signal (Real Part)

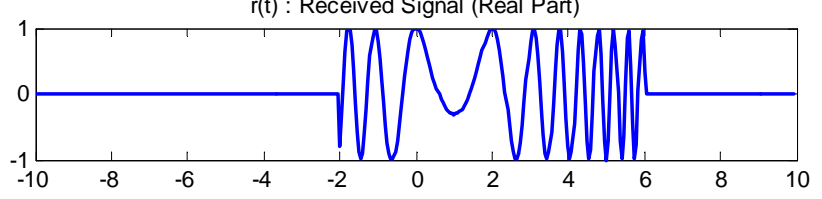

$\mathrm{A}_{\mathrm{r}, \mathrm{s}}(\tau, 0)$ : Matched Filter Output (Magnitude)

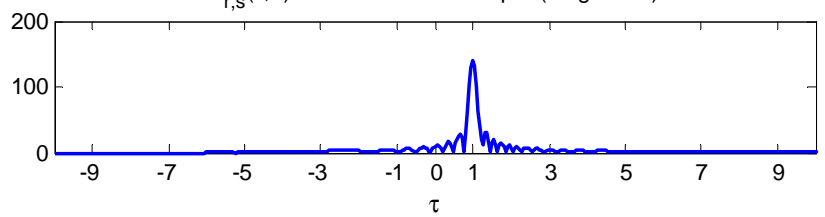

Fig. 3. Transmitted signal, received signal and the matched filter output. Signal is received at a delay of 2 seconds and has a Doppler frequency shift of $0.6 \mathrm{~Hz}$

The projection on the cross - ambiguity plane can be calculated from equation (7) through the fractional Fourier transforms of $r(t)$ and $s(t)$ at domain of $a=\frac{2}{\pi} \arctan (k)+1$ where $k$ is the chirp rate. Then a peak search is executed on the projection result. The location of the peak gives us the point of intersection of lines (I) and (II). The radial distance of the peak location to the origin is labeled as $d_{I}$ in Figure 4.

In the second stage of the search process, we calculate the slice of the ambiguity function along the line (II) using equation (6). To calculate the slice, the $a$ th order fractional Fourier transform of $r(t)$ and $s(t)$ is calculated where $a=$ $\frac{2}{\pi} \arctan (k)$. Note that the slice of cross ambiguity function along the line (II) is not a radial one. As explained in [5], the non-radial slice is calculated by a coordinate change on $r(t)$ and $s(t)$ :

$$
\begin{aligned}
d_{0} & =d_{I} / \sin (\arctan (k)) \\
\widetilde{r}(t) & =r\left(t+d_{0} / 2\right) \\
\widetilde{s}(t) & =s\left(t-d_{0} / 2\right)
\end{aligned}
$$

Here $d_{0}$ is the distance between the origin and the intersection point of line (II) and $\tau$ axis. (The parameter $d_{0}$ is also the peak location of the matched filter output.) The functions $\widetilde{r}(t)$ and $\widetilde{s}(t)$ are formed by delaying and advancing corresponding waveforms. The non-radial slice of the ambiguity function along the axis (II) can then be calculated using fractional Fourier transforms of $\widetilde{r}(t)$ and $\widetilde{s}(t)$ from equation (6) as in [5].

Figure 5 shows the result of the proposed algorithm on the presented example. The peak location given in the top panel of Figure 5 is the intersection of the lines (I) and (II). One can observe that the projection operation successfully localizes the LFM signal at the radial distance of $d_{I}=0.5257$.

In the second window of Figure 5, the non-radial slice of the ambiguity function is evaluated. As expected, the slice has 


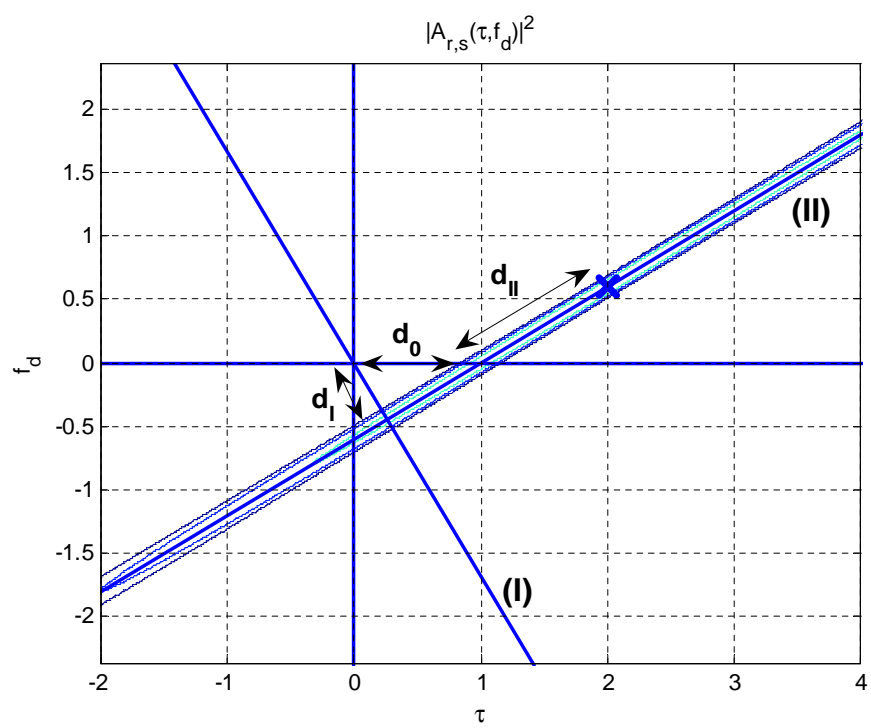

Fig. 4. Cross-ambiguity function for $r(t)$ and $s(t)$. The target location is shown by $\mathrm{X}$.

a triangular shape along this axis, since the chirp modulation is removed. The peak location in the slice indicates the target location. For the given example, the peak occurs at $d_{I I}=$ 1.176 .

Finally, the target delay and Doppler frequency is estimated using the following formulas:

$$
\begin{aligned}
\widehat{\tau} & =d_{0}+d_{I I} \cos (\arctan (k)) \\
\widehat{f_{d}} & =d_{I I} \sin (\arctan (k))
\end{aligned}
$$

For the given example, the target delay and Doppler frequency is estimated as 2.015 seconds and $0.6028 \mathrm{~Hz}$.
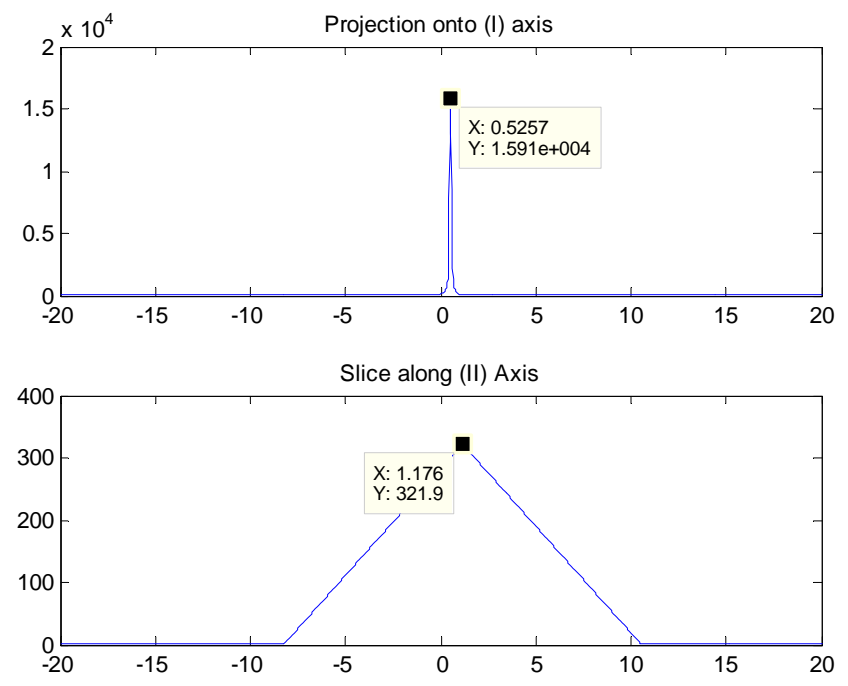

Fig. 5. Results of the proposed algorithm

On the Implementation. There are two methods available for the calculation of the fractional Fourier transform. The first method uses $\mathrm{O}(N \log N)$ operations and is an approximation to the fractional Fourier transform kernel, [12]. The approximation is based on the Nyquist theorem.

The second method generalizes DFT matrix to fractional DFT matrices, [13]. This method does not have a fast implementation therefore requires $\mathrm{O}\left(N^{2}\right)$ operations. Furthermore this method requires an a-priori eigenvector calculation which should be done offline.

For the presented example, we have used the fast method where the samples of $s(t)$ and $r(t)$ are formed in $[-10,10]$ with the sampling period of $1 / 20$ seconds. A total of 400 samples are collected for each signal.

\section{Conclusions}

We have presented an alternative method for the parameter estimation of LFM signals. The method uses the inherent structure of LFM signals in the ambiguity plane to reduce the 2-dimensional search required for ML detection to two 1dimensional search operations. The method is illustrated with an example.

The method can be attractive in the applications where the pulse duration is long. Such situations may arise in high Doppler resolution applications and also in sonar signal processing. The performance of the method under noisy conditions is to be examined at a later work.

\section{ACKNOWLEDGMENT}

Author would like to acknowledge the support of TUBITAK for this work under grant no. 106E187.

\section{REFERENCES}

[1] M. A. Richards, Fundamentals of Radar Signal Processing. New York: McGraw-Hill, 2005.

[2] R. Fitzgerald, "Effects of Range-Doppler Coupling on Chirp Radar Tracking Accuracy," IEEE Trans. Aerospace and Electronic Systems, vol. 10, no. 4, pp. 528-532, 1974.

[3] H. L. V. Trees, Detection, Estimation and Modulation Theory, Part III. New York: Wiley, 1968.

[4] M. Wang, A. K. Chan, and C. K. Chui, "Linear Frequency-modulated Signal Detection Using Radon-Ambiguity Transform," IEEE Trans. Signal Processing, vol. 46, no. 3, pp. 571-586, 1998.

[5] A. K. Ozdemir and O. Arikan, "Fast Computation of the Ambiguity Function and the Wigner Distribution on Arbitrary Line Segments," IEEE Trans. Signal Processing, vol. 49, pp. 381-393, February 2001.

[6] O. Akay and G. F. Boudreaux-Bartels, "Fractional convolution and correlation via operator methods and an application to detection of linear FM signals," IEEE Trans. Signal Processing, vol. 49, no. 5, pp. 979-993, 2001.

[7] O. Arikan and A. K. Ozdemir, "System and Method for Detection and Tracking of Targets," United States Patent 6636174, May 2007.

[8] B. K. Jennison, "Detection of Polyphase Pulse Compression Waveforms Using The Radon-Ambiguity Transform," IEEE Trans. Aerospace and Electronic Systems, vol. 39, no. 1, pp. 335-343, 2003.

[9] H. M. Ozaktas, The Fractional Fourier Transform with Applications in Optics and Signal Processing. England: John Wiley and Sons, 2001.

[10] A. K. Ozdemir, Novel Time-Frequency Analysis Techniques for Deterministic Signals. PhD thesis, Bilkent University, Ankara, Turkey, 2003.

[11] C. Stutt, "A Note On Invariant Relations For Ambiguity and Distance Functions," IEEE Trans. Information Theory, vol. 5, no. 4, pp. 164-167, 1959.

[12] H. M. Ozaktas, O. Arikan, M. A. Kutay, and G. Bozdagi, "Digital computation of the fractional Fourier transform," IEEE Trans. Signal Processing, vol. 44, no. 9, pp. 2141-2150, 1996.

[13] C. Candan, M. A. Kutay, and H. M. Ozaktas, "The discrete fractional Fourier transform," IEEE Trans. Signal Processing, vol. 48, no. 5, pp. 1329-1337, 2000. 